\title{
Adjuvant epidermal growth factor receptor tyrosine kinase inhibitors: are we actually ready?
}

\author{
Fabrizio Tabbò^, Maria Lucia Reale, Silvia Novello \\ Department of Oncology, San Luigi Gonzaga Hospital, University of Turin, Orbassano, Italy \\ Correspondence to: Fabrizio Tabbò. Department of Oncology, San Luigi Gonzaga Hospital, University of Turin, Orbassano, Italy. \\ Email: fabrizio.tabbo@unito.it. \\ Comment on: Liang W, Cai K, Chen C, et al. Society for Translational Medicine consensus on postoperative management of EGFR-mutant lung \\ cancer (2019 edition). Transl Lung Cancer Res 2019;8:1163-73.
}

Submitted Apr 09, 2020. Accepted for publication Apr 24, 2020.

doi: $10.21037 /$ tlcr-20-545

View this article at: http://dx.doi.org/10.21037/tlcr-20-545

Despite tremendous improvement in its clinical management, lung cancer is still the leading cause of cancer related death worldwide (1). It is usually diagnosed at advanced stage and only a minority of patients presents an early and potentially curable disease. However, with positive results of low-dose computed tomography (CT) screening in the high-risk population and with the improvement of diagnostic techniques, early diagnoses can hopefully increase in the near future (2). Therefore, defining the best curative treatment approach for this disease setting is of crucial relevance.

Liang and colleagues focused their attention on the subset of epidermal growth factor receptor (EGFR) positive patients cured with surgical resection and drew up a consensus on postoperative adjuvant targeted therapy management (3). As well known, EGFR mutations occur in the $10-15 \%$ of Caucasian patients and up to the $40 \%$ of Asian ones (4). EGFR testing is recommended in all patients with advanced non-squamous non-small cell lung cancer (NSCLC), but also in patients with pure squamous histology, especially if young and/or nonsmokers. EGFR tyrosine kinase inhibitors (TKIs) represent the recommended first-line treatment in patients with advanced NSCLC bearing classic mutations (i.e., exon 19 in-frame deletions and exon 21-point mutations) (5). Several randomized trials have demonstrated, indeed, the superiority of oral treatment with EGFR TKIs compared to standard platinum-based chemotherapy for this subgroup of patients (4). In the consensus document, authors stated that also NSCLC patients who have undergone a radical resection need EGFR mutation profiling and that EGFRTKIs can replace chemotherapy in patients candidate to adjuvant therapy after surgery, above all in patients who would probably not tolerate chemotherapy treatment, defining "at least 2 years" as the optimal treatment duration. In absence of direct comparisons, the reported adjuvant treatments are heterogeneous: chemotherapy, EGFR-TKI, chemotherapy plus EGFR-TKI (3).

Actually, several studies have evaluated the possibility of biological drugs as adjuvant therapy and the results are quite conflicting. What is clear is that, even in early stages, adjuvant treatment with EGFR TKIs could be effective only in those patients whose tumor expresses an EGFR activating mutation. Two are the phase III studies showing that erlotinib for 2 years or gefitinib for 1 year did not improve disease-free survival (DFS) [erlotinib: hazard ratio (HR) $0.90,95 \%$ confidence interval (CI): 0.74-1.10, $\mathrm{P}=0.32$; gefitinib: HR 1.28; 95\% CI: 0.92-1.76; $\mathrm{P}=0.14]$ or overall survival (OS) (erlotinib: HR 1.13, 95\% CI: 0.88-1.44, $\mathrm{P}=0.33$; gefitinib: HR 1.24; $95 \%$ CI: $0.90-1.71 ; \mathrm{P}=0.18$ ) compared to placebo in radically resected NSCLC patients (stage IB-IIIA) and not selected

$\wedge$ ORCID: 0000-0001-8525-2992. 
for EGFR mutations $(6,7)$. However, also in a molecular selected population, advantages seem limited only to DFS, which is a clear surrogate endpoint of efficacy especially in the adjuvant setting, and OS data remain immature. In two randomized trials, one phase II (the EVAN trial) and one phase III (the ADJUVANT study) conducted in Asian patients with EGFR-positive radically operated stage II-III NSCLC, a benefit in DFS was observed with erlotinib for 2 years (percentage of free disease patients: $81.4 \%, 95 \%$ CI: 69.6-93.1\% versus $44.6 \%$, 95\% CI: $26.9-62.4 \%$; $\mathrm{P}=0.0007)$ and with gefitinib for 2 years (28.7 versus 18.0 months, HR 0.60 ; $95 \%$ CI, $0.42-0.87, \mathrm{P}=0.005$ ), when compared to the adjuvant chemotherapy with cisplatin-vinorelbine $(8,9)$. In both studies, the OS advantage could not be demonstrated. A phase II study conducted in patients with surgicaltreated high-risk IIIA stage EGFR+ NSCLC reported an improvement in DFS (HR 0.37; 95\% CI: 0.16-0.85; $\mathrm{P}=0.014$ ), in the absence of OS benefit, with gefitinib for 6 months versus observation alone after adjuvant chemotherapy (10). Another phase II study, performed in resected patients with NSCLC (stage IB-IIIA) and activating EGFR mutation, did not report a significant increase in DFS with icotinib for 4-8 months versus the only observation after platinum-based adjuvant chemotherapy (11). Furthermore, Pennell et al. published a single-arm phase II trial of adjuvant erlotinib after surgery and consolidative chemo/ radiotherapy in stage IA-IIIA NSCLC patients, reporting a 2-year DFS of $88 \%$ (no median DFS or OS reported) (12). A recent metanalysis confirmed that adjuvant TKIs reduce the risk of recurrence in EGFR mutant NSCLC patients without benefit in OS (13). Trials with OS as primary endpoint such as the ALCHEMIST-EGFR (NCT02193282) study, in which EGFR+ NSCLC patients are randomized to 2-year erlotinib or placebo after adjuvant chemotherapy, are still ongoing (14). The absence of OS improvement is a critical aspect in evaluating the benefit of a treatment with adjuvant intent. Pignon et al. in 2008 published the Lung Adjuvant Cisplatin Evaluation (LACE) meta-analysis that included individual data from 5 phase III studies, which randomized patients with stage I-IIIA NSCLC radically operated to receive cisplatin-based chemotherapy versus observation only (15). This metaanalysis reports a HR of 0.89 (95\% CI: $0.82-0.96, \mathrm{P}=0.005$ ), which corresponds to an absolute 5 -year survival benefit of $5.4 \%$, in favor of adjuvant chemotherapy. Thus, in the absence of improved OS data for adjuvant EGFR TKIs compared to chemotherapy, at present, it seems reasonable to do not recommend routine molecular testing in the adjuvant setting, unless functional to the inclusion in clinical trials. Targeted therapies, with or without chemotherapy, cannot be recommended in the adjuvant treatment of NSCLC outside of experimental protocols (5).

Another element of concern for strong positive recommendations for adjuvant TKIs is related to the heterogeneity of trials, design, selected population and duration of treatment. The definition of a timeframe of 2-year treatment after surgical resection, as reported by authors, seems quite empiric. Thus, even if targeted therapies are usually more tolerated than chemotherapy, is important to underline that can be characterized by adverse events such as skin, gastrointestinal toxicities or fatigue for the entire duration of treatment (2 years versus 3 months of chemotherapy), potentially requiring dose reduction, treatment interruption and impairing patient compliance: actually in clinical trials up to one-third of patients did not complete the two years pre-planned treatment. Also, in the case of more tolerable treatment, as $3^{\text {rd }}$ generation TKI, the risk of relevant toxicities exists (e.g., lung interstitial disease), although low.

In the consensus document, authors propose to follow-up EGFR+ patients with annual brain magnetic resonance imaging (MRI) and bone scan, additionally to conventional CT, because of the higher risk of recurrence for this patients' subset (3). The European Society for Medical Oncology (ESMO) recommend the brain staging with MRI for all patients in stage III (16). For the other disease stages international recommendations are quite heterogeneous. The National Comprehensive Cancer Network (NCCN) recommends brain MRI in all patients with early or locally advanced NSCLC, excluding stage IA (5); the National Institute for Health and Care Excellence (NICE) recommends brain CT in stage II and brain MRI for people with stage III NSCLC who are having a treatment with curative intent (17); on the other hand the American College of Chest Physicians (ACCP) limits the indication to stage III and IV and in case of symptomatic patients (18). As well-known, NSCLC patients with EGFR mutations appear to have a higher incidence $(50-60 \%)$ of brain metastasis (19), therefore appears reasonable to implement brain-imaging follow up for their early detection. However, considering the absence of improvements in OS, solid prospective data are needed before recommending an intensive follow up strategy. Bone scintigraphy is considered optional in the imaging workup for diagnosis and staging by ESMO guidelines (16) and current data are still weak to implement its execution in case 
of asymptomatic patients.

Which should be the hypothetical best TKI to be used in the adjuvant setting is another element of uncertainty. Liang et al. in the consensus emphasize the role of osimertinib as the best option; nevertheless, available data of $3^{\text {rd }}$ generation TKI usage in EGFR+ patients are, currently, limited to stage IV patients with EGFR mutations (exon 19 deletion of L858R allele), for whom is preferable in respect to $1^{\text {st }}$ generation TKI, as elucidated in the FLAURA trial. Updated OS data reported a gain of 6.8 months of survival when osimertinib is administered upfront (HR for death, 0.80; 95.05\% CI: 0.64-1.00; $\mathrm{P}=0.046$ ) (20). For these reasons is plausible to consider its role at relapse for EGFR+ patients initially treated with surgery, but no evidence exists preferring the administration of osimertinib, rather than other EGFR TKI, in the adjuvant setting. The results of ongoing trial ADAURA (NCT02511106), expected in the third quarter of 2021, will give us relevant insights about the role of adjuvant osimertinib in terms of DFS-as primary endpoint-OS and health-related quality of life, as secondary endpoints (21).

Furthermore, unfortunately, we still ignore the precise biological mechanism at the basis of adjuvant EGFR targeting. We cannot exclude flare reactions with TKIs suspension, potentially caused by the reactivation of the microscopic disease that targeted therapies are unable to completely eradicate (22). And, more clinically relevant, we have no tools to forecast the biological evolution of EGFR+ neoplastic cells under drug pressure. If the rate of T790M emergence during adjuvant treatment with $1^{\text {st }}$ generation TKI seems low (12), we do not have any ideas whether treatment with more potent TKI might spur the development of more aggressive clones, difficult to be treated after; recent data shed light on relevant alternative mechanisms of resistance, such as histotype transition and off-target alterations that should be taken into account when an adjuvant treatment is selected (23).

Finally, the position paper on the postoperative management of EGFR-mutated NSCLC patients highlights a relevant aspect in thoracic oncology, adjuvant targeted treatments for EGFR+ patients, which will acquire even more significance in the next future of improved screening and early detection. However, the only availability of PFS data and the need of larger studies in not-Asiatic population put emphasis on the importance of having more solid and numerous data before recommending the use of EGFRTKIs in the adjuvant setting of our daily clinical practice.

\section{Acknowledgments}

Funding: None.

\section{Footnote}

Provenance and Peer Review: This article was commissioned by the editorial office, Translational Lung Cancer Research. The article did not undergo external peer review.

Conflicts of Interest: All authors have completed the ICMJE uniform disclosure form (available at http://dx.doi. org/10.21037/tlcr-20-545). SN has the following COI: Speaker Bureau/Advisor: Abbvie, AZ, Eli Lilly, Amgen, Roche, BMS, Takeda, Novartis, MSD, BI. SN serves as an unpaid editorial board member of Translational Lung Cancer Research from Jul 2019 to Jul 2021. The other authors have no conflicts of interest to declare.

Ethical Statement: The authors are accountable for all aspects of the work in ensuring that questions related to the accuracy or integrity of any part of the work are appropriately investigated and resolved.

Open Access Statement: This is an Open Access article distributed in accordance with the Creative Commons Attribution-NonCommercial-NoDerivs 4.0 International License (CC BY-NC-ND 4.0), which permits the noncommercial replication and distribution of the article with the strict proviso that no changes or edits are made and the original work is properly cited (including links to both the formal publication through the relevant DOI and the license). See: https://creativecommons.org/licenses/by-nc-nd/4.0/.

\section{References}

1. Bray F, Ferlay J, Soerjomataram I, et al. Global cancer statistics 2018: GLOBOCAN estimates of incidence and mortality worldwide for 36 cancers in 185 countries. CA Cancer J Clin 2018;68:394-424.

2. de Koning HJ, van der Aalst CM, de Jong PA, et al. Reduced Lung-Cancer Mortality with Volume CT Screening in a Randomized Trial. N Engl J Med 2020;382:503-13.

3. Liang W, Cai K, Chen C, et al. Society for Translational Medicine consensus on postoperative management of EGFR-mutant lung cancer (2019 edition). Transl Lung Cancer Res 2019;8:1163-73. 
4. Pirker R, Buder A, Filipits M. Osimertinib in advanced EGFR T790M-positive non-small-cell lung cancer: the clinical impact of AURA3. Transl Cancer Res 2017;6:S265-9.

5. Ettinger DS, Wood DE, Aisner DL et al. NCCN clinical practice guidelines in oncology: non-small cell lung cancer version 3.2020. February 11, 2020. Available online: https://www.nccn.org/professionals/physician_gls/pdf/ nscl.pdf (22 March 2020, date last accessed).

6. Kelly K, Altorki NK, Eberhardt WE, et al. Adjuvant Erlotinib Versus Placebo in Patients with Stage IBIIIA Non- Small-Cell Lung Cancer (RADIANT): A Randomized, Double-Blind, Phase III Trial. J Clin Oncol 2015;33:4007-14.

7. Goss GD, O'Callaghan C, Lorimer I, et al. Gefitinib versus placebo in completely resected non-small-cell lung cancer: results of the NCIC CTG BR19 study. J Clin Oncol 2013;31:3320-6.

8. Yue D, Xu S, Wang Q, et al. Erlotinib versus vinorelbine plus cisplatin as adjuvant therapy in Chinese patients with stage IIIA EGFR mutation-positive non-small-cell lung cancer (EVAN): a randomised, open-label, phase 2 trial. Lancet Respir Med 2018;6:863-73.

9. Zhong WZ, Wang Q, Mao WM, et al. Gefitinib versus vinorelbine plus cisplatin as adjuvant treatment for stage II- IIIA (N1-N2) EGFR-mutant NSCLC (ADJUVANT/ CTONG1104): a randomised, open-label, phase 3 study. Lancet Oncol 2018;19:139-48.

10. Li N, Ou W, Ye X, et al. Pemetrexed-carboplatin adjuvant chemotherapy with or without gefitinib in resected stage IIIA-N2 non-small cell lung cancer harbouring EGFR mutations: a randomized, phase II study. Ann Surg Oncol 2014;21:2091-6.

11. Feng S, Wang Y, Cai K, et al. Randomized Adjuvant Chemotherapy of EGFR-Mutated Non-Small Cell Lung Cancer Patients with or without Icotinib Consolidation Therapy. PLoS One 2015;10:e0140794.

12. Pennell NA, Neal JW, Chaft JE et al. SELECT: A Phase II Trial of Adjuvant Erlotinib in Patients With Resected Epidermal Growth Factor Receptor-Mutant Non-SmallCell Lung Cancer. J Clin Oncol 2019;37:97-104.

13. Raphael J, Vincent M, Boldt G, et al. Adjuvant Epidermal
Growth Factor Receptor Tyrosine Kinase Inhibitors (TKIs) in Resected Non-Small Cell Lung Cancer (NSCLC): A Systematic Review and Meta-analysis. Am J Clin Oncol 2019;42:440-5.

14. Alden RS, Mandrekar SJ, Oxnard GR. Designing a definitive trial for adjuvant targeted therapy in genotype defined lung cancer: the ALCHEMIST trials. Chin Clin Oncol 2015;4:37.

15. Pignon JP, Tribodet H, Scagliotti GV, et al. Lung adjuvant cisplatin evaluation: a pooled analysis by the LACE Collaborative Group. J Clin Oncol 2008;26:3552-9.

16. Postmus PE, Kerr KM, Oudkerk M, et al. Early and locally advanced non-small-cell lung cancer (NSCLC): ESMO Clinical Practice Guidelines for diagnosis, treatment and follow-up. Ann Oncol 2017;28:iv1-iv21.

17. Lung cancer: diagnosis and management NICE guideline Published: 28 March 2019. Available online: https://www. nice.org.uk/guidance/ng122 (22 March 2020, date last accessed).

18. Silvestri GA, Gonzalez AV, Jantz MA et al. Methods for staging nonsmall cell lung cancer diagnosis and management of lung cancer, 3rd ed: American College of Chest Physicians evidence-based clinical practice guidelines. Chest 2013;143:e211S-e250S.

19. Shin DY, Na II, Kim CH, et al. EGFR mutation and brain metastasis in pulmonary adenocarcinomas. J Thorac Oncol 2014;9:195-9.

20. Ramalingam SS, Vansteenkiste J, Planchard D, et al. Overall Survival with Osimertinib in Untreated, EGFR-Mutated Advanced NSCLC. N Engl J Med 2020;382:41-50.

21. Wu YL, Herbst RS, Mann H, et al. ADAURA: Phase III, Double-blind, Randomized Study of Osimertinib Versus Placebo in EGFR Mutation-positive Early-stage NSCLC After Complete Surgical Resection. Clin Lung Cancer 2018;19:e533-6.

22. Ng TL, Camidge DR. Lung cancer's real adjuvant EGFR targeted therapy questions. Lancet Oncol 2018;19:15-7.

23. Schoenfeld AJ, Chan JM, Kubota D, et al. Tumor Analyses Reveal Squamous Transformation and Off-Target Alterations As Early Resistance Mechanisms to First-line Osimertinib in EGFR-Mutant Lung Cancer. Clin Cancer Res 2020;26:2654-63.
Cite this article as: Tabbò F, Reale ML, Novello S. Adjuvant epidermal growth factor receptor tyrosine kinase inhibitors: are we actually ready? Transl Lung Cancer Res 2020;9(5):17241727. doi: $10.21037 /$ tlcr-20-545 